\title{
Die Europäische Union als Triebkraft der Privatisierung
}

Der zunächst gescheiterte Versuch, eine Verfassung für die Europäische Union (EU) zu etablieren, hat gezeigt, dass Krisentendenzen des Neoliberalismus auch die EU erreicht haben. Mit dem Reformvertrag von Lissabon wurden die Bedenken gegenüber der marktliberalen Verfasstheit der Europäischen Union weitgehend übergangen. Auch die durch die EU geförderte Liberalisierung und Privatisierung gerät durch Proteste auf lokaler und nationaler Ebene vermehrt unter Druck. Allerdings ändern diese Konflikte aufgrund der strukturellen Weichenstellung der „neuen europäischen Ökonomie“ nicht die grundsätzliche wettbewerbsorientierte Stoßrichtung der europäischen Institutionen. Welche Rolle spielt nun die EU bei Privatisierungsprozessen in Europa? Welche Entwicklungen zeichnen sich derzeit ab?

\section{Einleitung}

Analysen, die den Einfluss der Europäischen Union (EU) auf die Privatisierungspolitik der (westeuropäischen) Mitgliedsstaaten untersuchen, konzentrieren sich vor allem auf die Sektoren der netzgebundenen Infrastruktur: Telekommunikation, Post, Energie und Schienenverkehr sind sicherlich die am häufigsten durchleuchteten Sektoren in der Forschung zu europäischer Liberalisierungs- und Privatisierungspolitik. Die Europäische Kommission hat seit Ende der 1980er Jahre und verstärkt in den 1990er Jahren sektorale Liberalisierungsrichtlinien für diese Bereiche initiiert (Bieling/Deckwirth 2008a). Die Relevanz der europäischen Ebene für die großen Liberalisierungs- und Privatisierungsprojekte in den Netzwerk-Dienstleistungen ist somit kaum umstritten. Mittlerweile scheint allerdings der Höhepunkt der europäischen Liberalisierungspolitik in der netzgebundenen Infrastruktur erreicht zu sein. Mit Ausnahme des Wassersektors wurden in allen bedeutenden Infrastruktursektoren Sektorrichtlinien erlassen, sodass es sich bei der derzeitigen EU-Politik in diesen Bereichen vorrangig um Erweiterungen, Anpassungen oder Nachbesserungen handelt. Auf nationaler Ebene wurden die Richtlinien weitgehend umgesetzt und große Privatisierungsprojekte - etwa im Bereich der Telekommunikation - vollzogen (Privatization Barometer 2008, S. 3).

Hat also der Einfluss der EU auf die Privatisierungspolitik der Mitgliedstaaten nachgelassen? Keineswegs. Zum einen erweisen sich selbst die Erweiterungen und
Anpassungen im Bereich der netzgebundenen Infrastruktur als äußerst kontrovers. Insbesondere im Energiesektor liefern sich derzeit die Regierungsvertreter der Mitgliedstaaten heftige Auseinandersetzungen mit der EU-Kommission. Zum anderen wird der Einfluss der EU auf öffentliche Dienstleistungen im Bereich des Wettbewerbsrechts verstärkt ausgedehnt, indem neue Sektoren in die Binnenmarktbestimmungen integriert werden. Zudem sorgt auch die Herausbildung eines europäischen Finanzmarktkapitalismus dafür, dass die öffentliche Dienstleistungserbringung unter Privatisierungsdruck gerät.

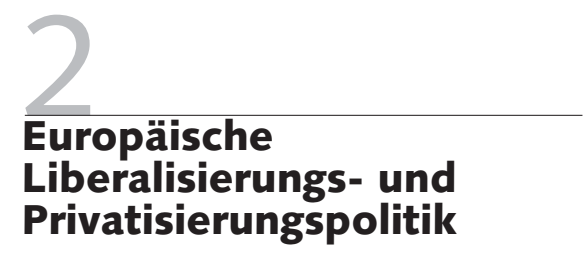

\subsection{KURZER HISTORISCHER ABRISS}

Seit Ende des 19. Jahrhunderts hat sich in großen Teilen Europas ein weit gespanntes Netz öffentlicher Dienstleistungen herausgebildet. Dazu zählten vor allem die Verund Entsorgungsdienste (Wasser und $\mathrm{Ab}$ wasser, Energie, Entsorgung), die Nachrichtenübermittlung (Fernmeldewesen, Post und Rundfunk), der Verkehrssektor, Banken sowie zahlreiche soziale und kulturelle Dienste (z. B. Bildung, Gesundheit, Altenpflege, Bibliotheken und Theater). Diese öffentlichen Dienstleistungen wurden insbesondere im Rahmen der westeuropäischen Wohlfahrtsstaaten der Nachkriegszeit unter keynesianischen Vorzeichen ausgebaut. Sie waren eingebettet in eine Politik des sozialen Ausgleichs, durch den die kapitalistische Dynamik zumindest teilweise eingehegt wurde.

In den Jahrzehnten nach dem Zweiten Weltkrieg wurden die nationalen Entwicklungsmodelle durch den Prozess der europäischen Integration zunächst abgestützt: Während die Außenwirtschaftspolitik der EG-Mitgliedstaaten bereits früh vergemeinschaftet wurde, blieb die nationale Souveränität in binnenwirtschaftlichen und sozialpolitischen Angelegenheiten weitgehend unangetastet. Dies galt auch für die öffentlichen Dienste. Zwar konnten sich Frankreich und Italien nicht mit ihren Forderungen nach einer weitgehenden Herausnahme des öffentlichen Sektors aus dem Vertrag der Europäischen Wirtschaftsgemeinschaft (EWG) von 1957 durchsetzen, dennoch bot der Vertrag die Möglichkeit, öffentliche Unternehmen neben privaten aufrecht $\mathrm{zu}$ erhalten. So stellte der EWG-Vertrag eigentumsrechtlich explizit die Neutralität der europäischen Institutionen fest (Art. 295 EGV), obwohl er auch für öffentliche Unternehmen grundsätzlich die gleichen Wettbewerbsbedingungen wie für private vorsah (Art. 86 EGV; Wimmer/Kahl 2001, S. 59). Der Kompromisscharakter des EWG-Vertrages bezüglich öffentlicher Unternehmen hatte zunächst zu einer fast vollständigen Inaktivität europäischer Institutionen geführt. Insgesamt konnte im

Christina Deckwirth, wissenschaftliche Mitarbeiterin am Institut für Politikwissenschaft an der Philipps-Universität Marburg. Arbeitsschwerpunkte: Europäische Integration, Internationale politische Ökonomie, Handels- und Privatisierungspolitik. e-mail: deckwirth@staff.uni-marburg.de 
Zeitraum von Ende der 1950er bis Mitte der 1970er Jahre die öffentliche Infrastruktur unter den Bedingungen eines hohen Wirtschaftswachstums weiter ausgebaut und gefestigt werden (Tsoukalis 1997, S. 20).

In der Folge der Wirtschaftskrise der 1970er Jahre wandelte sich in den 1980er Jahren dann jedoch die europäische Integrationsweise. Das Binnenmarktprojekt, das Mitte der 1980er Jahre entstand und im Jahr 1986 mit der Einheitlichen Europäischen Akte in europäisches Recht gegossen wurde, sollte die EU bis zum Jahr 1992 „fit für den Weltmarkt" machen (Sandholtz/ Zysman 1989). In diesem Kontext leitete die Europäische Kommission ein umfangreiches Liberalisierungs- und Deregulierungsprogramm ein. Darunter fiel - anders als in den Anfangsjahren der EG - erstmals nicht allein der Güterverkehr, sondern auch der Dienstleistungssektor. Die Wirtschafts- und Währungsunion, die mit dem Vertrag von Maastricht im Jahr 1992 eingeleitet wurde und schließlich im Jahr 2002 in die Einführung des Euros in zehn Mitgliedstaaten mündete, stärkte die gemeinsame europäische Währungs- und Finanzpolitik. Von größter Bedeutung waren in diesem Zusammenhang die Einrichtung der autonomen Europäischen Zentralbank (EZB) sowie die Konvergenzkriterien, die eine Politik der Austerität für öffentliche Haushalte vorgaben. In den Jahren 1999 und 2000 schließlich entstanden mit dem Financial Services Action Plan (FSAP) und der Lissabon-Strategie die beiden jüngsten großen wirtschaftlichen Integrationsprojekte. Während der FSAP die Finanzmarktintegration in der Europäischen Union fördern sollte, ist das zentrale Ziel der Lissabon-Strategie, die europäische Wirtschaft zu einer wissensbasierten Informationsgesellschaft auszubauen. Die dabei verfolgte marktliberale-monetaristische Ausrichtung impliziert, dass die europäische Integration nicht mehr der Absicherung nationaler Entwicklungsmodelle dient, sondern deren Umbau zu wettbewerbsorientierten Ökonomien aktiv vorantreibt.

\subsection{SEKTORALE RICHTLINIEN}

In diesem Kontext wandelte sich auch die europäische Position zu öffentlichen Dienstleistungen. Die europäische Liberalisierungspolitik begann im Telekommunikationssektor, der bis zu seiner Reorganisation in PTT-Behörden (Post, Telefon, Telegrafie) eingebunden war. Diese Struktur geriet seit den späten 1970er Jahren unter Druck. So setzten in diesem Zeitraum in den ostasiatischen Ländern ökonomische Aufholprozesse im Hoch-TechnologieMarkt ein und forderten damit die europäischen Anbieter heraus. Gleichzeitig bot sich der Telekommunikations-Sektor als neue Verwertungssphäre für anlagesuchendes Kapital an. Durch diese Entwicklungen gerieten die staatlichen PTT-Behörden als monopolistisch organisierte Anbieter unter Anpassungsdruck, da sie - so die sich ausbreitende marktliberale Position den Modernisierungsprozessen nicht gewachsen seien.

Die Europäische Kommission reagierte auf diese Veränderungen, indem sie erste europäische Liberalisierungs-Richtlinien initiierte, die folgende Elemente enthielten: Regelungen zum Marktzugang für Wettbewerber, die Einrichtung einer unabhängigen Regulierungsbehörde sowie die Vorgabe der Trennung bzw. „Entflechtung“ hoheitlicher und wirtschaftlicher Aufgaben. Neben dieser marktschaffenden Liberalisierung und Wettbewerbs-Regulierung enthielten die späteren Richtlinien auch marktkorrigierende Regulierungen. Letztere waren in dem Konzept des Universaldienstes enthalten, das Minimalstandards für die Universaldienstleistungserbringung, d.h. ein flächendeckendes Angebot, eine hohe Qualität der Dienstleistungen sowie spezifische Anforderungen des Nutzerund Verbraucherschutzes, vorsah. Sinkende Endkunden-Preise nach der Liberalisierung und Privatisierung der Telekommunikation schufen schließlich die Legitimationsgrundlage für weitere Richtlinien oder wie es Daniel Seikel (2008, S. 75) formuliert: „Die Liberalisierungspolitik wurde beim Bezahlen sinkender Telefonrechnungen hegemonial.“

Die Telekommunikations-Richtlinie lieferte die grobe Vorlage für weitere Richtlinien im Energiesektor (1996, 1998, 2003), im Postsektor $(1997,2002)$ sowie im Schienenverkehr (1991, 1994, 2001). Die Erarbeitung der sektoralen Richtlinien für die netzgebundene Infrastruktur avancierte so zu einem zentralen europäischen Projekt der 1990er Jahre. Es wird bis heute fortgeführt. Im Ergebnis wurden diese Sektoren in den europäischen Mitgliedstaaten liberalisiert, wobei sowohl sektoral als auch regional noch zahlreiche Unterschiede fortbestehen. Während die Liberalisierung des Telekommunikationssektors schon weit fortgeschritten ist und annähernd alle frü- heren staatlichen Telekommunikationsunternehmen (teil-)privatisiert wurden, bestehen im Schienensektor weiterhin starke Wettbewerbsbeschränkungen. Im Energiesektor wurde durch zwei sogenannte Beschleunigungsrichtlinien für Elektrizität und Gas die Liberalisierung nach anfänglich schwachen Wettbewerbsvorgaben weiter verschärft. Seit jüngster Zeit wird auf europäischer Ebene verstärkt über die eigentumsrechtliche Entflechtung diskutiert. Im Postsektor schließlich ist nach mehrfacher Verschiebung die vollständige Marktöffnung nun für das Jahr 2011 vorgesehen.

In zahlreichen europäischen Ländern vor allem dort, wo die Liberalisierung mit einer strategischen Förderung von nationalen Champions einherging - sind mittlerweile starke transnationale Infrastrukturkonzerne entstanden, wie etwa die Deutsche Telekom oder die spanische Telefónica im Telekommunikationssektor, E.on, EDF, Endesa oder Vattenfall im Energiesektor oder die Deutsche Post und die niederländische TNT im Post- und Logistiksektor (vgl. Bieling/Deckwirth 2008b). Allerdings sind die meisten dieser großen transnationalen Infrastrukturkonzerne aus den früheren staatlichen Monopolisten hervorgegangen. Neue Wettbewerber konnten sich vermehrt allein im Mobilfunkbereich auf dem Markt etablieren - doch auch hier handelt es sich meist um Töchter der früheren staatlichen Unternehmen. Der angestrebte Wettbewerb im europäischen Infrastrukturmarkt hat sich also nur begrenzt eingestellt. So erweisen sich die großen Infrastruktur-Unternehmen zugleich als Triebkräfte wie auch als Bremsblöcke bei der Formulierung der europäischen Liberalisierungspolitik. Schließlich konnten sie nicht nur von der Marktöffnung im europäischen Ausland profitieren, sondern prosperierten auch durch industriepolitische Maßnahmen zur Förderung nationaler Champions im Heimatland.

Die Rolle der EU bei der Liberalisierung und Privatisierung öffentlicher Dienstleistungen beschränkt sich jedoch keineswegs auf die sektoralen Richtlinien in der netzgebundenen Infrastruktur. Vielmehr lassen sich zwei weitere europäische Einflüsse ausmachen, die vor allem in den letzten Jahren wichtiger wurden: Erstens wird im Kontext der Binnenmarktintegration auch das europäische Wettbewerbsrecht auf horizontaler Ebene weiter auf den Bereich der öffentlichen Dienstleistungen ausgedehnt. 
Zweitens bestehen strukturelle politökonomische Einflussfaktoren - vor allem im Rahmen der Finanzmarktintegration -, die die nationale und lokale Liberalisierungsund Privatisierungspolitik systematisch begünstigen.

\section{Die Ausweitung des europäischen Wettbewerbsrechts}

In welchem Verhältnis stehen nun öffentliche Dienstleistungen und das europäische Wettbewerbsrecht im Rahmen des gemeinsamen Binnenmarktes? Hierbei handelt es sich keinesfalls um eine juristische Spitzfindigkeit, sondern um eine höchst politische Frage. Das europäische Wettbewerbsrecht für den Binnenmarkt beinhaltet neben dem Kartellrecht und der Fusionskontrolle auch Regelungen zu staatlichen Beihilfen. Das sind beispielsweise Subventionen oder Steuernachlässe sowie Bestimmungen zur öffentlichen Vergabepolitik (Art. 86-88 EGV). Bei diesen Maßnahmen handelt es sich um zentrale staatliche Steuerungsinstrumente, durch die etwa sozialpolitische Belange (Vergünstigungen für bestimmte soziale Gruppen im öffentlichen Personen-Nahverkehr (ÖPNV)), umweltpolitische Aspekte (Förderung des ÖPNV gegenüber dem Straßenverkehr) oder öffentliche Rechtsformen gegenüber privaten Trägern bei öffentlichen Ausschreibungen gefördert werden können. Eine solche Sonderbehandlung öffentlicher Unternehmen ist Kernbestandteil des Wirtschafts- und Sozialmodells einer Mixed Economy. Die Ausweitung des europäischen Wettbewerbsrechts in diesen Bereichen hat zur Folge, dass die gemischtwirtschaftlichen Entwicklungsmodelle der Mitgliedstaaten unter Druck geraten.

\subsection{SCHUTZ VERSUS INTEGRATION DER DASEINSVORSORGE}

Schon bei der Erarbeitung der Römischen Verträge (1957) entzündeten sich Kontroversen an der Stellung öffentlicher Unternehmen im europäischen Recht. Mit dem Binnenmarktprogramm gewann die Frage nach der Anwendbarkeit des Wettbewerbsrechts wieder an Bedeutung. Neben der übergeordneten politisch-normativen Frage nach der Funktion staatlicher Marktein- griffe besteht seither in zwei Bereichen konkreter Klärungsbedarf. Erstens wird diskutiert, welche Bereiche und Sektoren überhaupt unter die Bedingungen der europäischen Wettbewerbspolitik fallen bzw. welche Ausnahme- und Sonderregelungen für die Sektoren der Daseinsvorsorge ${ }^{1}$ gelten. Zweitens bestehen Unklarheiten bei der Präzisierung und Implementierung des europäischen Wettbewerbsrechts in den Bereichen, die für die öffentlichen Dienstleistungen von besonderer Relevanz sind, d.h. vor allem staatliche Beihilfen einschließlich des Vergaberechts.

Grundsätzlich stehen sich in der Debatte zwei Positionen gegenüber, die historisch jeweils von unterschiedlichen Akteuren vertreten wurden. Einerseits gibt es das Bestreben, die öffentliche Daseinsvorsorge weitestgehend aus dem Regelungsumfang der EU herauszunehmen. Diese Position zum Schutz der Daseinsvorsorge nahm ihren Ursprung in der Unsicherheit kommunaler Akteure bei der Anwendung des europäischen Wettbewerbsrechts auf die lokalen Infrastrukturleistungen im Zuge der Umsetzung des Binnenmarktprogramms. Kommunale Interessenverbände - unterstützt durch Regierungsvertreter aus Belgien und Frankreich - drängten schon seit Mitte der 1990er Jahre auf eine Klärung der europarechtlichen Stellung öffentlicher Dienstleistungen. Diese Initiativen standen zunächst im Kontext von Jacques Delors' Initiative zur Stärkung des „sozialen Europas“, die auf eine tiefe EU-Skepsis infolge der sozio-ökonomischen „Post-Maastricht-Kri$\mathrm{se}^{\text {“ }}$ reagierte (Rodriguez 1998). Einen ersten Erfolg erreichten die Befürworter eines rechtlichen Schutzes für öffentliche Dienstleistungen mit der Integration des Artikels 16 in den Vertrag von Amsterdam im Jahr 1997, in dem die besondere Bedeutung der Daseinsvorsorge für die Werte der Europäischen Union hervorgehoben wurde. Dieser Artikel brachte zwar kaum substanzielle rechtliche Veränderungen, wurde allerdings dennoch als diskursive Verschiebung und damit als Erfolg in der Auseinandersetzung um die Stärkung der Daseinsvorsorge angesehen. Darüber hinaus erstellte die Kommission in den Jahren 1996 bis 2003 erste Vorschläge für eine Rahmenrichtlinie zum Schutz der Daseinsvorsorge. Nachdem diese allerdings von zahlreichen Wirtschaftsverbänden massiv kritisiert wurden, verschwand dieses Vorhaben zwischen 2004 und 2006 weitestgehend von der europäischen Agenda und galt als gescheitert. Seit dem Jahr 2006 setzen sich allerdings der Europäische Gewerkschaftsbund (EGB), die Europäischen Gewerkschaften für den öffentlichen Dienst (EGÖD) sowie die sozialdemokratische Fraktion im Europäischen Parlament erneut für eine Rahmenrichtlinie zum Schutz der öffentlichen Dienste ein.

Die Debatte um den Schutz der Daseinsvorsorge wurde andererseits seit etwa dem Jahr 2000 zunehmend durch Liberalisierungsbestrebungen überlagert. Mit dem Ziel, die EU zum „wettbewerbsfähigsten, dynamischsten wissensbasierten Wirtschaftsraum der Welt" zu machen, sollte eine Strategie zur Liberalisierung des noch „unterentwickelten“ Dienstleistungssektors erarbeitet werden (Europäischer Rat 2000). Nachdem die Liberalisierung anderer Netzwerkindustrien bereits fortgeschritten war, erschien die Ausdehnung der wettbewerbsorientierten Reorganisation auf weitere Sektoren wie eine natürliche Folge. Für diese Position der Integration der Daseinsvorsorge in das europäische Wettbewerbsrecht machten sich vor allem die europäischen Wirtschaftsverbände wie UNICE (seit 2007: Business Europe), Eurochambres oder der BDI ${ }^{2}$ stark.

\subsection{NEUE WEICHENSTELLUNGEN}

In den Jahren 2005 und 2007 wurden nun drei neue Weichen gestellt, die sowohl die Position Schutz der Daseinsvorsorge als auch die Position Integration der Daseinsvorsorge aufgriffen:

(1) Im Jahr 2005 erfolgte eine Präzisierung der Art und Weise, wie das europäische Beihilferecht auf öffentliche Unternehmen angewendet wird (Europäische Kommission 2005a). Angestoßen durch mehrere EuGHUrteile bewertete die Kommission darin Beihilfen für kommunale Unternehmen bis

\footnotetext{
1 Für jene Dienstleistungen, die im deutschen Sprachgebrauch häufig als Daseinsvorsorge bezeichnet werden, wird in der europäischen Rechtssprache der Begriff der Dienstleistungen im allgemeinen Interesse verwendet. Diese werden im Interesse der Allgemeinheit erbracht und sind daher mit spezifischen Gemeinwohlverpflichtungen verknüpft.

2 UNICE steht für Union des Conféderations de l'Industrie et des Employeurs d'Europe, d.h. Union der Industrie- und Arbeitgeberverbände Europas. Eurochambre ist der europäische Dachverband der Industrie- und Handelskammer; BDI steht für den Bundesverband der deutschen Industrie.
} 
zu einem gewissen Maße als vertragskonform und berücksichtigte damit die besondere Stellung der „Dienstleistungen im allgemeinen Interesse“. Allerdings wurde der Spielraum der Mitgliedstaaten für die Vergaben von Beihilfen bzw. Ausgleichszahlungen massiv eingeengt. So sind nun etwa nachträgliche Zahlungen, mit denen finanzielle Verluste ausgeglichen werden sollen, oder nachträgliche Vorschriften für die Art und Weise der Erbringung der Dienstleistungen nur noch in Ausnahmefällen zulässig (Krajewski 2007, S. 446). Zudem verlangt die Kommission einen erheblichen administrativen Aufwand bei der Vergabe von Beihilfen. Dies bedeutet für die öffentlichen Unternehmen, dass ihre Finanzierungsmöglichkeiten eingeschränkt werden und sich dadurch der Druck zu (Teil-)Privatisierung und Ausgliederung von unrentablen Unternehmen erhöht (Löwe 2003).

(2) Die besondere Stellung der „Dienstleistungen im allgemeinen Interesse" wurde als Protokoll in den Reformvertrag von Lissabon aus dem Jahr 2007 integriert. ${ }^{3}$ Darin werden die gemeinsamen Werte der Union für diese Dienste benannt: das Subsidiaritätsprinzip, soziale und kulturelle Verschiedenartigkeiten sowie ein hohes $\mathrm{Ni}$ veau bezüglich der Qualität, Sicherheit, Bezahlbarkeit, Gleichbehandlung und des universellen Zugangs (Europäische Kommission 2007, S. 10). Hieraus lassen sich durchaus Ansatzpunkte für die Überprüfung bestehender sektoraler Richtlinien sowie Anforderungen an neue Richtlinien ableiten. Auch die Grundrechte-Charta erkennt die nationale Zuständigkeit für die Gewährleistung des Zugangs zu Dienstleistungen der netzgebundenen Infrastruktur an. $\mathrm{Ob}$ diese Bezüge im Reformvertrag allerdings die Ausweitung wettbewerbsorientierter Regelungen aufhalten können, erscheint äußerst fraglich.

(3) Schließlich erarbeitete die Kommission im Jahr 2007 eine Mitteilung unter dem Titel „Dienstleistungen von allgemeinem Interesse unter Einschluss von Sozialdienstleistungen “. Darin stellt die Kommission fest, dass „abgesehen von den Tätigkeiten im Zusammenhang mit der Ausübung öffentlicher Gewalt [...] die überwiegende Mehrheit der Dienstleistungen als, wirtschaftliche Tätigkeiten' im Sinne der Binnenmarktvorschriften des EG-Vertrages [...] zu betrachten sind." (Europäische Kommission 2007, S. 6). Insbesondere die sozialen Dienste und die Gesundheitsversorgung werden ausdrücklich als neue „Blickpunkte“ bei der Anwendung des europäischen Wettbewerbsrechts genannt. Diese Formulierungen sind in mehrerer Hinsicht äußerst brisant. Zum einen verfügt die EU kaum über Kompetenzen im Bereich der sozialen Dienste. Zudem galt es zuvor als ungeklärt, ob soziale und Gesundheitsdienste zu den ,nicht-wirtschaftlichen Dienstleistungen“ zählen, die von den Wettbewerbsbestimmungen im Binnenmarkt ausgenommen sind. Noch bei der Erarbeitung der sogenannten Bolkestein-Richtlinie wurden aufgrund massiven Protests die sozialen Dienste und die Gesundheitsdienste aus dem Geltungsbereich ausgenommen. Die neue Mitteilung stellt somit den Versuch dar, die sozialen und Gesundheitsdienste durch die Hintertür in die Binnenmarktbestimmungen zu integrieren.

In diesen drei Initiativen spiegelt sich der grundsätzliche Widerspruch, aber auch die unmissverständlich wettbewerbsorientierte Stoßrichtung der EU in Fragen der Daseinsvorsorge wider: Auf der einen Seite stellen die Kommission und der Europäische Rat wiederholt die zentrale Bedeutung der „Dienstleistungen im allgemeinen Interesse“ für das europäische Sozialmodell fest und verankern dieses sogar im Reformvertrag. Auf der anderen Seite greift das Wettbewerbsrecht zunehmend auf weitere - mitunter äußerst sensible - Sektoren über. Damit erweist sich die wiederholte Betonung der Sonderstellung der Dienstleistungen im allgemeinen Interesse als trügerisch. In der Praxis bedeutet dies, dass sich öffentliche Unternehmen oder freie Träger, die Dienste der Daseinsvorsorge erbringen, in Konkurrenz zu privaten Unternehmen auf dem Markt behaupten müssen. Sie unterliegen damit ebenso dem Zwang zur Kostenreduzierung und dem Druck zur Steigerung von Umsatz und Gewinn, während die Orientierung auf Universaldienste und das Allgemeinwohl relativ an Bedeutung verlieren. Rationalisierungen - vor allem im Bereich der Beschäftigten - sowie veränderte betriebliche Abläufe und Expansion ins Ausland sind die Folge (Löwe 2003, S. 193). Eine Privatisierung solch kommerziell agierender Unternehmen erscheint dann folgerichtig. Neben den bereits europarechtlich liberalisierten Sektoren der netzgebundenen Infrastruktur könnten von diesen Prozessen der Kommerzialisierung und Privatisierung zukünftig vor allem sensible Bereiche wie die Wasserversorgung, die Abfall- und Abwasserentsorgung, der ÖPNV sowie die Kinderbetreuung, die Altenpflege und die Gesundheitsdienste betroffen sein.

\section{Europäischer Finanzmarkt- kapitalismus}

Zusätzlich zum Binnenmarkt und der Ausweitung des Wettbewerbsrechts fördert auch der strukturelle Wandel der europäischen Ökonomie die Privatisierung. In den letzten Jahren haben dabei insbesondere die Finanzmärkte und die Finanzpolitik einen Bedeutungszuwachs erfahren (Huffschmid 2007). Dies hat vor allem zwei Gründe: Zum einen haben die Marktkapitalisierung der Unternehmen und der Aktienhandel enorm zugenommen. Dies ist nicht allein auf die kontinuierliche Liberalisierung und Deregulierung der Finanzmärkte zurückzuführen, sondern auch auf eine andauernde Umverteilung der Lohneinkommen hin zu den Vermögenseinkommen. Private Vermögensbesitzer befinden sich daher auf der Suche nach neuen renditeträchtigen Anlagefeldern. Zum anderen ist durch den Paradigmenwechsel zu einer angebotsorientierten monetaristischen Wirtschaftspolitik seit Mitte der 1980er Jahre eine Austeritätspolitik hegemonial geworden, deren vornehmliche Ziele die Bekämpfung der Inflation und ein ausgeglichener Staatshaushalt sind.

\subsection{DIE ENTSTEHUNG EINES EUROPÄISCHEN FINANZMARKTES}

Die Europäische Union hat diese Entwicklungen durch mehrere Maßnahmen gefördert. Als maßgeblich für die Einführung monetaristischer Prinzipien hat sich das Projekt der Wirtschafts- und Währungsunion (WWU) erwiesen. Dieses hatte vorrangig zum Ziel, die Geld- und Finanzpo-

3 Das Protokoll entstand vor dem Hintergrund eine Rechtsstreits zwischen der Europäischen Kommission und den Niederlanden um sozialen Wohnungsbau. Um ähnliche Konflikte zu vermeiden, drängte die niederländische Regierung vor allem auf das Subsidiaritätsprinzip und machte die Annahme des Protokolls zu einer Bedingung für ihre Unterschrift unter den Reformvertrag. 
litik der EU-Mitgliedstaaten zu disziplinieren, um so wiederum die europäische Wettbewerbsfähigkeit zu stärken. Eines der wichtigsten Ergebnisse war die Festlegung der sogenannten Konvergenzkriterien, durch deren Erfüllung sich die Mitgliedstaaten für den WWU-Beitritt qualifizieren mussten. Nach ihrer Überführung in den Stabilitäts- und Wachstumspakt im Jahr 1997 schreiben die Kriterien weiterhin vor, die staatliche Neuverschuldung (max. $3 \%$ des Bruttoinlandsprodukts (BIP)) sowie die Schuldenstandsquote (max. $60 \%$ des BIP) niedrig zu halten. Darüber hinaus wurde die Geldpolitik der Europäischen Zentralbank auf das übergeordnete Ziel der Inflationsbekämpfung festgelegt (Gill 1998). Diese vertraglichen Bestimmungen hatten eine dämpfende Wirkung auf das europäische Wirtschaftswachstum und folglich auch auf die Steuereinnahmen (Huffschmid 2007, S. 217ff.). Verstärkt wurden diese Tendenzen durch einen wachsenden Steuerwettbewerb zwischen den EU-Mitgliedstaaten. Vor allem in den Bereichen Finanzvermögen, Kapitaleinkünfte, Spitzen- und Unternehmensteuern konkurrieren die Mitgliedsländer um den besten Standort für Investoren. Diese Standortkonkurrenz hat sich durch die Osterweiterung zusätzlich verschärft. Gleichzeitig fehlen der europäischen Ebene die Möglichkeiten, steuernd in die Entwicklung der Beschäftigung und des Wirtschaftswachstums einzugreifen, da die EU nur über sehr geringe Haushaltsmittel verfügt und die Beschäftigungspolitik allenfalls lose koordiniert. In diesem Kontext dienten Einnahmen aus Privatisierungserlösen der kurzfristigen Konsolidierung angespannter öffentlicher Haushalte. Der Höhepunkt der Privatisierungen in Europa wurde Mitte der 1990er Jahre erreicht, d.h. in der Phase, in der sich die EU-Mitgliedstaaten bemühten, die Konvergenzkriterien zu erfüllen.

Die Veräußerungen fanden zunächst noch vorwiegend im Inland statt, da die europäischen Finanzmärkte noch wesentlich über die nationale Ebene reguliert waren. Dies änderte sich allerdings durch den wachsenden Einfluss der EU im Bereich der Finanzmärkte. Schon das Binnenmarktprogramm von 1985 hatte sich die Liberalisierung der europäischen Finanzmärkte zum Ziel gesetzt. Erst vor dem Hintergrund des Booms auf den Finanzmärkten im Kontext der New Economy rückte die Finanzmarktintegration in das Zentrum europäischer Integrationsbestre- bungen (Bieling 2003). Mit dem Financial Services Action Plan (FSAP) aus dem Jahr 1999 hatte sich die EU zum Ziel gesetzt, die europäischen Finanzmärkte bis zum Jahr 2005 weitgehend zu liberalisieren. Im Ergebnis wurden in den Mitgliedstaaten die traditionellen Beziehungen zwischen Haushalten und Unternehmen auf der einen und Banken auf der anderen Seite durch marktbasierte Strukturen abgelöst. Eigentumstitel und Kreditgeschäfte werden nun vermehrt als Wertpapiere - d.h. in Form von Aktien und Anleihen - an der Börse gehandelt. Dies betraf auch die öffentliche Infrastruktur, sofern diese privatisiert und von Unternehmen oder institutionellen Anlegern ganz oder teilweise erworben wurde.

\subsection{DER AUFSTIEG DER PRIVATE EQUITY FONDS}

Nach Ablauf der ersten Umsetzungsfrist des Finanzmarkt-Aktionsplans nahm die Europäische Kommission im Jahr 2005 einen erneuten Anlauf, um die Finanzmarktintegration weiter voranzutreiben (Europäische Kommission 2005b). Neben der Konsolidierung der bereits veranlassten Liberalisierungs- und Deregulierungsschritte betonte sie insbesondere die Notwendigkeit, die Märkte für Investitionsfonds weiter auszubauen. Als Gründe für diese Initiativen benennt die Kommission die strukturellen Veränderungen auf den europäischen Finanzmärkten und die „immer älter werdende Bevölkerung", was „mehr Verantwortung für ihre langfristigen Finanzierungsbedürfnisse" verlange (Europäische Kommission 2006, S. 2). Damit wird eine Stoßrichtung der Finanzmarktintegration benannt: Die Altersversorgung soll im Zuge der Umstrukturierung der umlagefinanzierten Rentensysteme vermehrt auf dem Kapitalmarkt erspart werden (ebd., S. 4). Darüber hinaus strebt die Kommission den Ausbau des Marktes für Privatplatzierungen an, d.h. vor allem für die weitgehend unkontrollierten und auf Kurzfristigkeit orientierten Private Equity Fonds (PEF) und Hedge Fonds. Um diese Prozesse voranzutreiben, richtete die Kommission eigene Sachverständigengruppen ein. Während es gängige Praxis ist, solche Beratungsgremien mehrheitlich mit Vertretern der Privatwirtschaft zu besetzen, ist es doch ungewöhnlich, dass die genannten Gruppen allein aus Vertretern einzelner Finanzunternehmen bestehen. ${ }^{4}$
Nachdem die großen staatlichen Infrastrukturunternehmen durch Börsengänge privatisiert worden sind, gewinnen derzeit die Private Equity Fonds in Privatisierungsprozessen an Bedeutung (Hall 2006; Levantini 2007). Zwar ist die Anzahl der Verkäufe öffentlicher Unternehmen, an denen PEF beteiligt waren, im Zeitraum zwischen 2002-2005 zurückgegangen, allerdings sind die Werte dieser Privatisierungen stark gestiegen. Sowohl McKinsey als auch die Financial Times identifizieren die weitere Privatisierung öffentlicher Unternehmen, einschließlich der Infrastruktur, als wichtige zukünftige Aktivitäten für Private Equity Fonds (Huffschmid 2007, S. 10). Von diesen Entwicklungen ist bereits eine ganze Bandbreite von Sektoren betroffen (Hall 2006, S. 225ff.): Private Krankenhäuser, die sich neu gegenüber ihren öffentlichen Konkurrenten am Markt etablieren, sind das Ziel von Risikokapital-Anlagen. Im Bereich der netzgebundenen Infrastruktur beteiligen sich die PEF vermehrt an bereits privatisierten Unternehmen, wie etwa an der Deutschen Telekom oder dem niederländischen Entsorgungsunternehmen AVR. Allerdings sind die Fonds auch direkt in Privatisierungsprozesse involviert, so beispielsweise in Form von Infrastrukturfonds im Bereich Energie, Wasser, Häfen oder Straßen. In Deutschland hat sich der Wohnungssektor als lukratives Anlagefeld erwiesen (Huffschmid 2007, S. 226f.; Vogtländer 2007). Das hat vor allem zwei Gründe: In diesem Bereich gibt es - im Vergleich zur netzgebundenen Infrastruktur - weniger staatliche Regulierungen. Zudem befindet sich die öffentliche Wohnungswirtschaft meist in der Hand der Kommunen oder der Bundesländer, wo der Druck zur Haushaltssanierung besonders hoch ist. Zwischen den Jahren 2003 und 2008 waren PEF an mindestens zehn Privatisierungen von großen Wohnungsbeständen sowie zahlreichen weiteren Immobilienkäufen beteiligt.

Diese Entwicklungen sind äußerst folgenreich für die Dienste der Daseinsvorsorge: Die wachsende Kapitalmarkt-Orientierung verstärkt die Ausrichtung auf kurzfristige Strategien der Profitmaximierung, um die Interessen der Anteilseigner zu bedienen (Shareholder Value). Diese Orien-

\footnotetext{
4 Vgl. http://ec.europa.eu/internal_market/
} investment/other_docs/index_en.htm\#reports. 
tierung schafft Instabilitäten und erhöht die Krisenanfälligkeit der betroffenen Unternehmen. Insbesondere ein Ausbau der Investitionsmöglichkeiten für die weitgehend unkontrollierten Private Equity Fonds verstärkt diesen Effekt. Für die Dienste der Daseinsvorsorge ist dies besonders brisant, da die Erbringung von Universaldiensten sowie sozialpolitische Funktionen - wie etwa im Wohnungssektor - gefährdet werden könnten.

\section{Perspektiven}

Die bisherigen Privatisierungen haben kaum die angestrebten Effekte der Effizienzund Qualitätssteigerung erreicht (Dickhaus/Dietz 2005). Zumindest auf kommunaler und zum Teil auch auf nationaler Ebene häufen sich daher die Proteste gegen Privatisierungsprojekte in Europa. Im Post- und Bahnsektor waren vor allem Gewerkschaften wichtige Akteure in Auseinandersetzungen um die Art und Weise der Restrukturierung der nationalen Infrastruktur. Auf kommunaler Ebene - etwa in den Bereichen Wasser- und Energieversorgung, Krankenhäuser oder Wohnungen bilden sich zunehmend Bürgerinitiativen heraus (Hall et al. 2005). Seit dem europäischen Sozialforum in Athen im Jahr 2006 haben sich soziale Bewegungen auch auf europäischer Ebene zusammengeschlossen, um sich europaweit für den Erhalt öffentlicher Dienste einzusetzen (Marcon/ Zola 2007, S. 15f.). Gleichzeitig engagiert sich der Europäische Gewerkschaftsbund (EGB) gemeinsam mit dem Europäischen Gewerkschaftsverband für den öffentlichen Dienst (EGÖD) für den Schutz öffentlicher Dienste durch die EU. Die europäischen Institutionen und Kräfteverhältnisse bleiben von diesen Prozessen nicht gänzlich unberührt. So konnte eine geplante Liberalisierungs-Richtlinie für den Wassersektor nicht realisiert werden, weil vor allem kommunale Interessensverbände, aber auch die Mehrheit im europäischen Parlament, eine verstärkte Privatisierung der Wasserversorgung befürchteten (Deckwirth 2008). Auch in den Auseinandersetzungen um die Bolkestein-Richtlinie spielten die öffentlichen Dienste eine Rolle - und wurden im Ergebnis aus der endgültigen Fassung herausgestrichen.
Allerdings handelt es sich hierbei kaum um tiefere Krisen in der europäischen Liberalisierungspolitik. Denn sowohl die Liberalisierung des Wassersektors als auch die Integration der sozialen Dienste in das Wettbewerbsrecht wurden zwar zunächst aufgehalten, aber schließlich auf anderem Weg fortgesetzt. Zudem sind es vor allem die strukturellen Faktoren der neuen europäischen Ökonomie, d.h. die verordnete Austeritätspolitik sowie die Finanzmarktintegration, die auch jenseits der großen Liberalisierungsprojekte der 1990er Jahre Privatisierungsprozesse in den Mitgliedstaaten befördern. Diese eher indirekte und noch wenig berücksichtigte Rolle der EU für die nationale und kommunale Privatisierungspolitik ist zwar weniger auffällig, dafür aber umso tückischer. Für soziale Bewegungen und Gewerkschaften bietet sie kaum Angriffsmöglichkeiten, um Kampagnen zum Erhalt öffentlicher Dienste zu organisieren. Die Proteste werden also weiter auf nationaler und kommunaler Ebene organisiert werden oder verstärkt die Kontextbedingungen der europäischen Privatisierungsprozesse thematisieren müssen.

\section{LITERATUR}

Bieling, H.-J. (2003): Social Forces in the Making of the New European Economy: The Case of Financial Market Integration, in: New Political Economy 2, S. 203-224

Bieling, H.-J./Deckwirth, C. (2008a): Die Reorganisation der öffentlichen Infrastruktur in der Europäischen Union - Einleitung, in: Bieling, H.-J./ Deckwirth, C./Schmalz, S. (Hrsg.): Liberalisierung und Privatisierung in Europa. Die Reorganisation der öffentlichen Infrastruktur in der Europäischen Union, Münster, S. 9-33

Bieling, H.-J./Deckwirth, C. (2008b): Privatising public infrastructure within the EU: the interaction between supranational institutions, transnational forces and national governments, in: Transfer 2, S. 237-257 Deckwirth, C. (2008): Sonderfall oder Hoffnungsträger? Die Privatisierung der Wasserversorgung in der Europäischen Union im Kontext der wettbewerblichen Reorganisation der öffentlichen Infrastrukturdienstleistungen, in: Wasserkolloquium (Hrsg.): Wasser. Die Kommerzialisierung eines öffentlichen Gutes, Berlin, S. 102-119
Dickhaus, B./Dietz, K. (2005): Öffentliche Dienstleistungen unter Privatisierungsdruck. Folgen von Privatisierung und Liberalisierung öffentlicher Dienstleistungen in Europa, WEED-Arbeitspapier, Berlin Europäische Kommission (2003): Grünbuch zu Dienstleistungen von allgemeinem Interesse, $\operatorname{KOM(2003)} 270$ endgültig, Brüssel

Europäische Kommission (2005a): Gemeinschaftsrahmen für staatliche Beihilfen, die als Ausgleich für die Erbringung öffentlicher Dienstleistungen gewährt werden. GD COMP/11//D(2005) 179, Brüssel Europäische Kommission (2005b): Weißbuch zur Finanzdienstleistungspolitik für die Jahre 2005-2010, KOM(05) 629 endgültig, Brüssel

Europäische Kommission (2006): Weißbuch für den Ausbau des Binnenmarktrahmens für Investmentfonds, KOM (2006) 686 endgültig, Brüssel Europäische Kommission (2007): Dienstleistungen von allgemeinem Interesse unter Einfluss der Sozialdienstleistungen: Europas neues Engagement, KOM (2007) 725 endgültig, Brüssel

Europäischer Rat (2000): Schlussfolgerungen des Vorsitzes. Europäischer Rat 23. und 24. März 2000, Lissabon/Brüssel 
Gill, S. (1998): European Governance and New Constitutionalism: Economic and Monetary Union and Alternatives to Disciplinary Neoliberalism in Europe, in: New Political Economy 1, S. 5-27

Hall, D. (2006): Private equity and infrastructure funds in public services and utilities, London

Hall, D./Lobina, E./de la Motte, R. (2005): Public resistance to privatisation in water and energy, in: Development in Practice 3/4, S. 286-301 Huffschmid, J. (2007): Finance as driver of privatisation, in: Transfer 2, S. 209-236

Krajewski, M. (2007): Leistungen der Daseinsvorsorge im Gemeinschaftsrecht. Freier Wettbewerb oder öffentliche Aufgabe, in: Wagner, A./Wedl, V. (Hrsg.): Bilanz und Perspektiven zum europäischen Recht. Eine Nachdenkschrift anlässlich 50 Jahre Römische Verträge, Wien, S. 433-453

Levantini, A. (2007): The Role of Private Equity Funds in Global Privatizations, in: The PB Newsletter 6, S. 14-23

Löwe, J. (2003): Ökonomisierung der öffentlichen Wirtschaft und die EUWettbewerbspolitik, in: Harms, J./Reichard, C. (Hrsg.): Die Ökonomisierung der öffentlichen Wirtschaft: Instrumente und Trends, Baden-Baden, S. 13-17

Marcon, G./Zola, D. (2007): A European Union of the Peoples, in: Eurotopia 4, S. 13-16
McCreevy, C. (2007): Private Equity - Batting for Europe. Rede gehalten am 22.2. auf der Konferenz Mergermarket/Deal Drivers Ireland $M \& A$ Conference in Dublin, URL: download: http://europa.eu/rapid/ pressReleasesAction.do? reference $=$ SPEECH/07/94\&format=PDF\&aged $=$ 1\&language $=E N \&$ guilanguage $=$ en [11.6.2008]

Privatization Barometer (2008): Executive Summary, in: The PB Report 2007, S. 3-5

Rodriguez, S. (1998): Les Services Publics et le Traité d'Amsterdam, in: Revue du Marché Commun et de I'Union Européenne 414/1, S. 37-46 Sandholtz, W./Zysman, J. (1989): 1992: Recasting the European Bargain, in: World Politics 1, S. 95-128

Seikel, D. (2008): Die Liberalisierungs-Agenda der EU - Entstaatlichung öffentlicher Dienstleistungssektoren in Europa, in: Vorgänge 2, S. 70-81 Tsoukalis, L. (1997): The New European Economy Revisited, Oxford Vogtländer, M. (2007): Die Privatisierung öffentlicher Wohnungen, in: Wirtschaftsdienst 11, S. 748-753

Wimmer, N./Kahl, A. (2001): Die öffentlichen Unternehmen im freien Markt. Strukturreformen. Europarechtliche Rahmenbedingungen, Wien Windolf, P. (Hrsg.) (2005): Finanzmarktkapitalismus. Analysen zum Wandel von Produktionsregimen, in: Kölner Zeitschrift für Soziologie und Sozialpsychologie, Sonderheft 45, Wiesbaden 